s'écartent progressivement de bas en haut : ce fait tient à ce que les charges varjent proportionnellement au carré des vitesses. M. Krir a trouvé le moyen d'obtenir sur le papier du cylindre des inscriptions proportionnelles aux vitesses mêmes; pour cela, il a ingénieusement combiné le levier rectiligne du style avec un autre levier faisant un angle avec le premier, monté sur le même axe et dont le pivot d'articulation avec la bielle se développe sur la partie haute du cylindre dans une situation telle que les chemins parcourus aient des bras de levier à peu près proportionnels aux vitesses. Les lignes de vitesses sont alors régulièrement espacées, ce qui rend plus facile et plus rapide la lecture du résultat.

Appareil Parenty. - Dans une lettre adressée au Génie Civil et reproduite par celui-ci, M. Parenty, directeur de la Manufacture des Tabacs de Lille, fait remarquer qu'en 1899 il reçut de M. Bechmann, directeur du Service des eaux de l'Exposition universelle de Paris, la commande d'un appareil reposant exactement sur le même principe que l'appareil Krir et Mesnager et n'en différant que par un détail de construction. Cet appareil servit, pendant toute la durée de l'Exposition, à jauger le débit des fontaines lumineuses, et fut ensuite acquis par la Ville de Paris.

Le tube de Pitot, qui sert de principe à l'appareil de M. Parenty, ne diffère pas de celui de MM. Krit et Mesnager; ses indications se lisent également sur le siphon $\mathrm{MN}$ dont la partie supérieure contient un certain volume d'air comprimé.
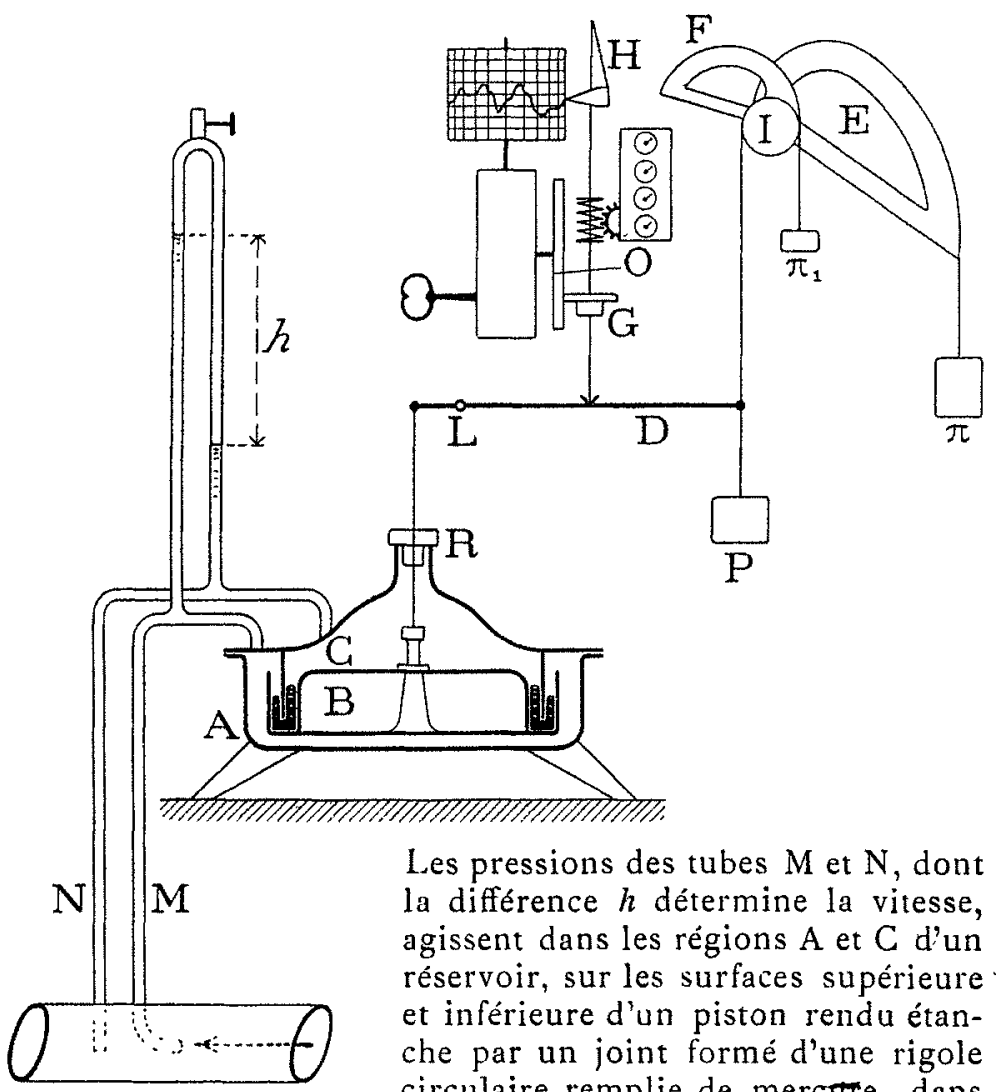

Les pressions des tubes $\mathrm{M}$ et $\mathrm{N}$, dont la différence $h$ détermine la vitesse, agissent dans les régions $\mathrm{A}$ et $\mathrm{C}$ d'un réservoir, sur les surfaces supérieure et inférieure d'un piston rendu étanche par un joint formé d'une rigole circulaire remplie de merctre, dans laquelle plonge un cylindre vertical relié au plafond supérieur de la région $C$.

Ce piston dont le diamètre est assez grand pour lui donner une force ascensionnelle déterminée, proportionnelle à $h$, est suspendu verticalement par un fil d'acier glissant dans un presse-étoupe garni de matière antifriction, par exemple, et pouvant se maintenir étanche pendant très longtemps, voire même pendant plusieurs années. Ce fil s'accroche à l'extrémité d'une balance de position qui peut être un simple levier coudé et enregistre, dans ce cas, la pression $h$ par des tangentes d'arc proportionnelles à $h$ elle-même.

M. Parenty a obtenu un résultat beaucoup plus satisfaisant au point de vue scientifique et industriel, en procurant au levier họizontal de suspension $D$ des déplacements dont les arcs soient rendus proportionnels, non pas à $h$, mais à $V \bar{h}$, cंest. à-dire à la vitesse et, par conséquent, au débit de la conduite. ll a suffi de suspendre le contrepoids terminus $\pi$ de la balance à une came $\mathrm{E}$, actionnée d'autre part par l'intermédiaire d'une poulie I, dont le brin vertical est accroché à l'extrémité du levier $\mathrm{D}$ opposée à la suspension du piston.

Le tracé de cette came est l'antipodère d'une spirale du second degré $: \rho=\rho_{1}+k \omega^{2}$.

Les bras de levier $\rho$ du contrepoids terminus $\pi$, proportionnels à $h$, vont ici croissant comme le carré des arcs ainsi rendus proportionnels à $/ \bar{h}$.

On peut observer que la plongée dans le mercure, du cylindre de métal tombant au plafond supérieur de la chambre C, oppose au mouvement ascensionnel du piston une résistance proportionnelle à la hauteur où s'élève ce piston, hauteur qui est de quelques millimètres seulement. Cette erreur est faible, et de plus elle peut être exactement compensée au moyen d'un second contrepoids $\pi_{1}$, suspendu sur une seconde came $F$ montée sur le même axe que la première $E$, et dont le tracé est l'antipodère d'une spirale du premier degré en $\omega$ (spirale $d^{\prime}$ Archimède) $: \rho=\rho_{1}^{\prime}+k \xi^{\prime} \omega$.

Cette disposition entièrement rationnelle fournit tous les avantages que l'on peut demander à un compteur de la vitesse et du débit des conduites forcées. D'après $M$. Parenty : $I^{\circ}[1$ est rigoureusement et mathématiquement exact. $2^{\circ} \mathrm{Il}$ est débarrassé des ressorts et flexibles dont la résistance est nécessairément variable et désagréable. $3^{\circ} \mathrm{Sa}$ sensibilité est indéfinie, car il est facile de donner au piston moteur un diamètre suffisant pour que son levier soit maintenu pendant la marche à l'état de de perpétuelle oscillation, et qu'il revienne très exactement au zéro pendant l'arrêt du courant. $4^{\circ}$ La totalisation des $V / \bar{h}$ exige absolument le tracé d'une courbe dont les ordonnées soient proportionnelles à cette grandeur $V$. Laire de la courbe, enregistrée par un style $\mathrm{H}$ sur un cylindre chronométrique, donne sans aucune erreur le débit total correspondant à une période quelconque. $5^{\circ}$ Le planimétrage de cette aire, c'est-à-dire la totalité des débits, s'effectue automatiquement et d'une manière très simple au moyen d'une petite roulette $G$ fixée sur l'axe vertical de soutien du style. Cette roulette se met en contact avec des circonférences du plateau chronométrique d'entrainement, de longueurs proportionnelles a l'abais. sement de cette roulette au-dessous du centre $O$, c'est-à-dire en définitive proportionnelles à $V \bar{h}$.

Les brevets et additions de brevets que M. Parenty a pris pour la construction de cette classe d'appareils comportent l'emploi d'une ou de plusieurs cuvettes contenant des liquides, eau ou mercure, dont les niveaux libres sont soumis à l'action dés pressions. Ces cuvettes peuvent être réunies entre ellể par des siphons ou par des flexibles, ou bien encore sont disposées concentriquement, de façon que l'une forme un piston mobile comme dans le cas présent.

Dans ces conditions, le seul changement ou perfectionnement apporté à l'appareil Parenty, se réduit ici à la substitution d'un peson à ressort à la balancerie de précision qu'il convient d'utiliser pour ces recherches délicates. L'appareil Parenty: Jauge des conduites forcées, fait d'aille urs partie des appareils de mesure auxquels l'Académie des Sciences a accordé le prix de Mécanique en 1896 .

\section{AVIS IMPORTANT}

\section{Le Bureau technique de LA HOUILLE BLANCHE} est transfèré, 24, rue Sully. Y adresser tout ce qui a lrait à la rédaction de la Revue. 


\section{LES GLACIERS DU DAUPHINÉ (1)}

\author{
Par M. W. KILIAN, \\ Prolesseur do Géologie a la Faculte des Scrences de Gremohlo
}

Le but de ces lignes n'est pas d'apporter de nouveaux éléments à la question de l'avenir des glaciers alpins, $n_{1}$ d'indiquer un moyen de ralentir ou d'empêcher une réduction dont nous constatons la marche inexorable et dont nous ignorons la durée ; nous pensons simplement farre œuvre utile en exposant l'état actuel de nos connarssances relativement aux glaciers dauphinors, en indiquant les principaux desuderata que suggère l'étude poursurvie depuis quatorze ans, dans les Alpes dauphinoises, par la Société des Touristes du Dauphiné et par la Commission frança1se des Glaciers ; enfin, en montrant aussı comment une connaissance méthodique et plus exacte de nos apparells glaciarres et de l'enneigement de nos montagnes peut épargner peut-être aux varllants ingénieurs qui tirent de la. " houille blanche " une énerg1e féconde pour le pays, des mécomptes fâcheux et facilement évitables.

\section{$*^{* * *}$}

Les glaciers du Dauphiné se répartissent en plusieurs groupes géographiques (v. plus loin) et présenient aussi des types assez varıés, bien qu'aucun d'entre eux ne possède l'amplitude des grands apparels glaciaires des Alpes suisses et autrichiennes. Ils ont fait dans ces dernières années l'objet d'études et d'observations assez suivies, dont il n'est pas sans intérêt d'envisager la marche et les résultats.

L'exploration pittoresque et topographique de nos Alpes, qui fut pratıquée avec tant d'ardeur et de succès par une plérade d'alpinistes pendant le dernier quart du XIX ${ }^{\mathrm{e}}$ siècle, quı fut vraiment l'époque héroıque de l'alpınısme, fit farre à lä connaissance, jusqu'alors incomplète, des Alpes françaises des progrès vraiment merveilleux.

Un des résultats les plus importants de ce mouvement, qui a pris depuis lors un caractère presque exclusivement sportif, et malheureusement de moins en moins compatible avec l'esprit scientifique, fut de montrer même aux observateurs les plus superficiels que nos glaciers, lom d'étre immuables dans leurs dimensions, étaient soumis à des changements considérables se traduisant tantôt par des crues, tantôt par des réductions notables, ainsi que l'attestent la comparaison de vues photographiques prises à différentes époques ou de cartes topographiques levées à des dates éloignées, ainsi que quelques documents historiques dont certains remontent jusqu'à la fin du XVI ${ }^{\circ}$ siècle.

Dès 1882 une de nos sociétés alpines les plus actives, la Société des Touristes du Dauphiné, s'était déjà préoccupée de l'ntérêt qui pouvait ressortir d'une observation attentive de ces variations. D'autre part, le professeur Forel, de Morges, publiait depuis une série d'années, dans l'Annuaire du Club Alpin suisse, des indications précieuses sur le mouvement de nos glaciers. Enfin, le prince Roland Bonaparte se livra, pendant les années I 889 à 1892 , avèc l'aide de quelques-uns de nos meilleurs guides de l'Orsans, à des travaux considérables de repérage et à une enquête sur le passé des principaux (34) glaciers dauphinois (v. $17^{\mathrm{e}}$ et $18^{\mathrm{c}}$ Annuaires du Club Alpin français, I 890 et $18 \mathrm{gI}$ ).

A partir de 1890 et sur l'initiative de deux professeurs de la Faculté des Sciences de l'Université de Grenoble, la Société des Touristes du Dauphiné organisa un service d'observations méthodiques, qui se continue encore à l'heure actuelle ét qui s'étendit aussi à l'enneigement de nos montagnes I es résul-

(1) Cet artucle a paru sous la signature de M. KILIAN, dans le livre Grenoble et le Dauphiné, qui a été offert aux congressistes de l'As. sociation Française pour l'Avancement des Sciences. M. KILTAN a bien voulu nous autoriser à le reproduire in extenso. tats, la méthode et l'historique de ces études, poursuivies pendant dix années et s'appuyant sur les recherches antérieures de M. Forel et du prince Roland Bonaparte, firent l'objet d'une série de rapports insérés dans les Annuaires de la Société, et furent réums, ęl rgor, en une publication importante (I), illustrée de nombreuses planches, et publiée avec le concours de l'Assocjation françarse pour l'Avancement des Scrences; nous en extrayons ce qui suit

"Vingt-six des principaux glaciers dauphinois ont été, de la part de notre Société, l'objet d'observations suivies pendant ces dix dernières années, cette enquête n'ayant pas pu embrasser la totalıté des glacıers qui auraient été de nature à présenter quelque intérêt ; on trouvera du reste dans les rapports annuels publies par la Société des Touristes du Dauphiné des ręnseignements isolés sur une foule d'autres glaciers.

"Sur ces vingt-six glaciers, étudiés d'une façon spéciale, un certain nombre de ceux sur lesquels existent des observations antérieures à 1892 ont subi, dans leur gronde phase de. décrue, datant, suivant les cas, d'une époque comprise entre I 858 et i 870 , un arrêt de leur mouvement de recul (glaciers du Lac et du Vallon, dans le massif de la Meije, versant Nord) et même une période de crute passagère (crue de fin du Xix" siècle, de M. Forel : glaciers du Rateau, de la Meije, du Monêtıer, du Casset, du Sélé), pendant une période comprise, suivant les cas, de 1889 à 1893 . Ces derniers glaciers sont silués sur les versants Nord et Nord-Est des massils rle la Meije et de Séguret-Foran, sauf le glacier du Sélé qui regarde l'Est.

"A l'exception du Glacier Blanc (versant Sud-Est du Pelvoux) et du glacier du Casset (versant Nord-Est du massif de Séguret-Foran), qui semblent encore stationnrires, tous les glaciers en observation sont aujourd'hui en décrue manifeste. Les glaciers de la région mérudionale du massif du Pelvoux (glaciers du Valsenestre et du Valjouffrey), en particulier, accusent une réduction telle que leur disparition complète est à craindre pour un avenir peu élongné; il en est de même du glacier Lombard, au Nord de la Romanche, dont le bassin d'alimentation est peu étendu. Les glaciers des Grandes-Rousses se signalent également, mais à un moindre degré, par leur décrue constante. Cependant, un gonflement précurseur d'une crue prochaine se produit actuellement pour trois glaciers (Chardon, Bonne-Pierre, Pllatte), appartenant tous au cirque du Vénéon.

"Le Glacier Blanc (versant Sud-Est du Pelvoux), l'un des plus grands de la région, s'est toujours singularisé par le défaut de concordance de ses phases avec celles des autres glaciers du Pelvoux. "En crue jusqu'en 1865, il a décru de I 865 à I 886 , est entré en crue depuis celte dernière date et a contınué jusqu'en I 899 une croissance qui n'a été que passagère pour cinq de ses voisins et ne s'est pas manifesté pour les autres (crue de fin du XIX ${ }^{\theta}$ sic̀cle, de M. Forel). Il serait curieux de rechercher les causes de ce régime spécial, si différent de celui du Glacier Noir, pourtant si proche du Glacier Blanc.

Ces résultats concordent, dans leurs grands traits, avec ceux qui ressortent des dernières études sur les variations des glaciers suisses, notamment avec ceux qui sont portés sur la carte de M. Forel, publiée dans le i $8^{\circ}$ rapport sur les Variations périodiques des glaciers des Alpes, en 1897 , rédigé par MM. F.-A. Forel, E. Lugeon et M. Muret $(A n n$. du C.A.S., t. XXXIII, I898), et avec les données mentionnées dans le IO ${ }^{\circ}$ rapport (1898), des mêmes auteurs ( $A n n$. du C. A. S., t XXXIV, I899).

"Dans les Alpes dauphinoises, ainsi que dans les Alpes suisses, il est beaucoup de glaciers qui n'ont pas subi la crue de fin du XIX siècle et, chez ceux qui l'ont manifestee, la durée de cette phase a été très variable. S'il y a encore chez nous, comme dans toutes les Alpes, quelques retardataires de cette crue de fin du XIX ${ }^{\circ}$ siècle, la grande majorité de nos glaciers est́, ici comme ailleurs, en phase manifeste de décrue. Cepen

(1) Voir plus loin la liste bibliographique. 
dant le gonflement observé (v. plus haut) chez trois glaciers du bassin du Vénéon peut être interprété comme l'indice précurseur d'un prochain changement de régime qui pourra s'étendre plus tard à d'autres glaciers.

"Ces faits suggèrent la réfiexion que les variatzons des glaciers, buen que semblant obéir à des lois générales, sont loin de se produire avec un synchronisme de détail rigoureux dans les appareils glaciarres d'un même massif. En outre, l'exposition de chacun d'eux ne semble pas avoir une influence exclusive sur ces divergences de détail, dont les causes paraissent être complexes et tenir à un ensemble de conditions locales d'ordre à la fors topographique et météorologique, notamment à la nature de leur bassin de réception.

" Il semble également que, depurs le milieu du siècle, les crues constatées, même celle sı considérable du Glacier Blanc, ne soient que des accidents ou des retards dans le phénomène général de décrue des glaciers alpins ( 1 ) $n$.

Ajoutons qu'un grand nombre de bonnes volontés individuelles, telles que celles de plusieurs guides, parmi lesquels nous citerons : J.-B. Rodier, de la Bérarde ; M.-F. Arnaud, de Barcelonnette, et André Antoıne, de Maurın, avaient concouru à tournir les documents de cette enquête. La publication de photographies datées augmentait la valeur des renseignements ainsi réunis.

Sur l'initiative des Congrès géologiques internatıonaux, une Commission mternationale des Glacuers s'est constituée depuis un certain nombre d'années (1897) ; cette Commission, dont l'auteur de ces lignes fait partie depuis 1900 , centralise les documents relatıfs aux variatıons des glaciers du monde entier; son but est de rechercher les loıs quı règlent ces variatıons ; elle a déjà fait paraître plusieurs rapports, assez succincts en ce qui concerne le Dauphiné.

La création, en juillet IgoI, d'une Commission françazse des Glaciers (I) donna plus d'ampleur et plus de suite à l'enquête si patiemment conduite par la Société des Tourıstes du Dauphiné. Cette Commission, placée sous le patronage simultané de cette dernière Société et du Club Alpin français, se compose actuellement de MM. le prince Roland Bonaparte, président d'honneur de la Commission internationale des Glaciers, président; Joseph Vallot, durecteur de l'Observatoire du Mont-Blanc, vice-président du Club Alpın français, viceprésident ; Charles Rabot, membre de la Commission centrale de la Socıété de Géographie de Paris, secrétaire ; Kilian, professeur à la Faculté des Sciences de l'Université de Grenoble, membre du bureau de la Société des Touristes du Dauphıné, rapporteur ; général Arvers, commandant la $28^{\mathrm{e}}$ divisıon d'infanterie à Chambéry ; Emile Belloc, ancien président de la Société de Pisciculture ; Marcelin Boule, professeur au Muséum d'Histoire naturelle ; Henry Cuënot, ancien élève de l'Ecole polytechnique, maître de conférences à la Faculté de Droit de l'Université de Paris ; Martel, secrétaire général de la Société de Spéléologie, Puiseux, professeur adjoint à la Faculté des Sciences de l'Université de Paris, vice-président du Club Alpin français ; Sauvage, ingénieur en chef des Mines, professeur à l'Ecole nationale des Mines ; Schrader, géographe, présıdent du Club Alpin françars ; Tavernier, ingénieur en chef des Ponts et Chaussées, à Lyon ; Henri Vallot, ingénieur à Paris ; Ch. Vélain, professeur à la 'Faculté des Sciences de l'Université de Paris ; Em. de Margerie, ancıen président de la Société géologique de France, directeur

(1) Les massifs si dénudés du haut bassin de l'Ubaye (massifs du Chambeyron, de Panestrel, etc) montrent, avec la dernière évidence, la trace d'une puissante extension, récente et considérable, d'appareils glaciaires, dont les glaciers de Marinet ne sont, en quelque sorte, qu'un témoin résiduel. Ces derniers présentent même, depuis deux ans, une telle diminution que l'on peut prévoir leur compléte disparition (Observations de IgoI)

(1) Voir le programme daction de cette Commission, reproduit dans le tome XXVII (1901) de l'Annuaire de la Société dés Touristes au Dauphine. des Annales de Géographie ; Flusın, Offner et Jacob, préparateurs à la Faculté des Sciences de Grenoble.

La Commission française des Glaciers a déterminé la créa. tion d'une collection de vites photographiques soigneusement datées et repérées, qui constıtuera pour l'avenir une source inestimable de documents comparatifs ; cette collection est, en ce quı concerne les Alpes, déposée au Laboratorre de Géolo gie de l'Université de Grenoble. Elle a déjà fait paraître, de puis sa récente fondation, outre de précieuses "Revues de glaciologie ", rédigées par M. Rabot, plusieurs rapports intéressant notre région

C'est ainsı qu'en 1901, MM. Kilian, Offner et Flusın publièrent des observations nouvelles sur les glaciers du Lac, de la Grrose, du Vallon, du Rateau, de la Meije, de l'Homme, du Monestier du Casset, de la Bonne-Pierre, du Chardon, de la Pılatte, du Sélé, du Glacier Blanc, du Glacier Noır, de Séguret et Foran (Eychauda), et sur les appare1ls glaciarres, connu sous le nom de glaciers de Marinet, desquelles 11 résulte que le recul est devenu général sur lous ces glaciers, sauf peut-être au glacier de la Bonne-Pierre ; les glaciers du Marinet, en particulier, présentent une déchéance remarquable et se trou vent dans une phase de diminution très rapide. Des vues photographiques documenta1res accompagnaient ce rapport.

En 1902, la Commission française des Glaciers chargea I Paul Girardin d'une campagne d'observations dans les Alpes de Savoie et du Dauphiné. En ce qui concerne notre champ d'études, M. Girardin s'est occupé des glaciers des Quirlies du Grand-Sablat et de celui de la Selle, et a constaté un recul considérable, surtout au glacier de la Selle, dont le front, depuis trente ans, aurait rétrogradé de 1200 mètres.

Ën I 893, des subventions accordées à la Commission françaıse des Glaciers par la Société des. Tourıstes du Dauphiné, par le Club Alpin françaıs, par l'Association française pour l'avancement des Sciences et par le prince Roland Bonaparte permirent d'effectuer dans les Alpes du Dauphiné une campagne d'études très sérieuse, qui fut confiée, sous la direction de l'auteur de ces lignes, à MM Flusin, Jacob et Offner, pré. parateurs de chimie, de géologie et de botanıque, à la Faculté des Sciences de Grenoble. Nous extrayons du rapport préliminaire, rédigé par M. Jacob, Agrégé des Sciences naturelles. sur cette mission d'études, les lignes sulvantes :

Cette exploration, qui permettra de donner une description détaillée de nombreux glaciers non encore observés, a été combinée de manière à visiter les deux versants de l'arête SudOuest du massif du Pelvoux qui, en s'étendant de la Roche de la Muzelle au massif des Bans, sépare la vallée du Vénéon du Valjouffrey et du Valgaudemard (I) Située à l'une des latitudes les plus méridionales où l'on observe des glaciers dans les Alpes françaıses, la régıon parcourue devait fournir, en cas de retrait, des indications particulièrement nettes. A ce sujet, les résultats obtenus sont péremptoires; le mouvement de recul est très accentué dans les glaciers observés. Ceux du versant Sud (glacier du Grand et du Petit Vallon, d'Olan, du Lauzon, de Gioberney) ne sont plus que des névés sans importance occupant les angles rentrants de la chaîne, alors que des traces très fraîches et les indications recueillies sur place permettent d'affirmer qu'à une époque relativement récente, ces glaciers avaient l'extension que leur attribue la carte de l'EtatMajor. Le retrait a même amené la disparition complète de la glace sur les pentes exposées au Sud-Est : les glaciers de Porteras et de la Roche ont cessé d'exister. Les glaciers du versant Nord de la chaîne ont encore en revanche un grand développement, quoiqu'ils manifestent, eux aussi, un recul sensible. La partie inférieure de presque tous les glaciers de cette catégorie, en particulier des glaciers de la Mariande d'Entrepierroux, des Sellettes, du Fond de la Muande, se

(1) Consulter la carte topographique de l'Ftat-Major au i/80 000 feuille Briançon, ou mieux la carte du massif du Pelvoux au I/too o0o de M. H. Duhamel, Grenoble, 1892 . 
termine au-dessus de barres transversales de roches moutonnées, sans accumuler de blocs de glace en aval de celles-ci, comme le feraient des glaciers dans un stade d'avancée. De plus, dans les cirques dont l'ensemble offre à la fois les deux orientations vers le Nord-Ouest et le Nord-Est, - glaciers de la Mariande, d'Entrepierroux et du Says, - les glaciers véritables, avec séracs jalonnant les ruptures de pente, ne subsistent que sur les versants exposés au Nord-Ouest, tandis que les pentes orientées vers le Nord-Est ne sont plus recouvertes par la glace, mais montrent des barres successives, récemment mises à nu, de roches moutonnées séparant des névés étagés les uns au-dessous des autres.

"Les différences d'orientation ne suffisent pas seules à expliquer les faits observés; la nature du profil des vallées intervient aussi pour régler le régime de la diminution des glaciers. Sur les versants de la chaîne étudiée la pente n'offre pas de véritable concavité à l'origine des vallées, mais se trouve constituée par des gradins successifs étagés depuis l'arête jusqu'à la vallée proprement dite ; par suite de ce fait, il n'y a pas de grands réservoirs de neige pour alimenter le glacier et le retrait a pu faire sentir plus immédiatement ses effets, amenant la disparation des glaciers du versant Sud, rédusant les glaciers du versant Nord à de simples séracs el même fragmentant ceux-ci en une succession de névés sur les pentes exposées au Nord-Est. Ce régime est tout à fait différent de celui des grands glaciers situés plus à l'Ouest - glacuers du Chardon et de la Pllatte, Glacier Noir, Glacier Blanc. Ic1, la vallée présente à l'origine une ou plusieurs grandes cuvettes susceptibles de permettre l'accumulation de réserves de neige et de glace et çes glaciers sont, à l'heure actuelle, les moins atteints du massif du Pelvoux.

"D'après l'examen des repères antérieurement placés, le glacier du Chardon diminue d'épaisseur ; le front du glacier de la Pilatte a reculé, en moyenne, de 14 mètres depuis octobre Igor. Le front dù glacier des Etançons se modifie et recule surtout à l'Ouest (glacier des Etançons proprement dit) ; la glace n'occupe plus qu'une très mince bande en avant du promontoire de la Meije qui se trouve à la veille de séparer complètement le glacier du Pavé du glacier des Etançons proprement dit.

"Dans le massif des Grandes-Rousses, le front du glacier des Quirlies a, d'après M. Flusin, reculé de 3 où 4 mètres depuis septembre Igo2.

"Au cours de la campagne qui a fourni les résultats précédents, de nombreuses photographies documentaires, dont les clichés seront déposés au laboratoire de géologie de l'Université de Grenoble, ont été prises de points repérés. "

Un rapport détaillé et documenté, dont ces lignes ne sont qu'un parfait résumé, est actuellement sous presse ; il est illustré de photographies et de schémas très instructif́s.

La Commission française des Glaciers s'est en outre assuré pour IgO4 le concours du Ministère de l'Agriculture et de l'Administration des Forêts.

On voit, par ce qui précède, que les observations recueillies dans ces dernières années (I) démontrent avec évidence la

(1) On consultera notamment à ce propos :

XVII Kilian, Neige et glaciers (Ann. $S . T$. D., t. XVI, XVII, XVIII, XX).

W. Kilian et G. Flusin, Obscrvations sur les variations des glaciers et lenneigement dans les Alpes dauphinoises, organisées et subventonnées par la Société des Touristes du Dauphiné, de isgo à 1890 , et publiées sous le patronage de 1'Association trançaise pour lavancement des Scuences (Grenoble, Allier, 1900225 pp., 9 planches). Cet ouvrage conticnt les indications biographiques antérieures à 2000 .

W. Kilian et G. Flusin, Etudes glaciaires (avec 2 pl.) Annales Univ. Grenoble, t. XII, p. $88_{4}$ (1900)

W. Kilian, Rapport sur les variations des glaciers français de 1900 à I90I, présenté à la Commission française des glaciers (Ann.C.A. $F$, W.

W. Kilian, G. Flusin et J. Offncr, Nouvelles observations sur les et $A n_{n}$. $S T$ auphiné et de la Haute-Ubaye (Grenoble, Allier, rgor, et $A n n . S . T . D .$, t. XXVII, et $C . A . F .$, t. XXVIII, IgoI). diminution importante que subissent actuellement nos glaclers et qui va jusqu'à avoir considérablement modifié, depuis une quarantaine d'années, l'aspect de bon nombre de paysages de haute montagne. Cetle perte de la glaciation si notable en Dauphiné a été constatée d'une façon encore plus accentuée et évaluée dans d'autres parties des Alpes ; on l'a également signalée dans diverses régions montagneuses, par excmple dans les Andes (I).

L'annonce de la décrue actuelle qui semble menacer dans leur existence certains de nos glaciers dauphinois n'a pas été sans occasionner un intéressant échange de vues entre les glacialistes.

M. Forel a fait paraître au début de son $23^{\circ}$ rapport sur les varıations périodiques des glaciers des Alpes, publié en collaboration avec MM. M Lugeon et E. Muret (Annuaire du $S . A$. C. t. XXXVIII) un remarquable article dans lequel il s'attache à combattre l'idée, émise par nous, d'une disparition ultıme, dans un avenir, du reste indéterminé, des glaciers des Alpes et en particulier des glaciers du Dauphiné. Ce traval nous suggère les observations suivantes :

Si nous nous plaçons au point de vue géologique, nous no voyons pas de raison pour admettre la durée éternelle des glaciers alpins, alors que plusieurs massifs montagneux (Vosges, Karpathes, etc ) ont vu disparaître, il y a longtemps déjà, leur couronne glacée. Nous sommes loin cie nier la probabilité et la récurrence de crues importantes, mais de moins en moins considérables, pouvant survenir avant la destruction finale, surtout pour les glaciers des Alpes centrales, et nous nous bornons à affirmer que les glaciers du Dauphiné sont plus près de leur disparition que ceux des autres parties de la chaine.

On est du reste quelque peu surpris de voir, dans un probleme où Il s'agit d'échéances aussi lointaines, M. Forel se préoccuper de l'influence que pourraient avoir sur l' "Industrie des étrangers " en Suisse la réduction et la disparition des apparells glaciaires Quoi qu'il en soit, nous lui devons un exposé magistral dans lequel il essaye de répondre d'unc façon négative à la question : les glaciers des Alpes vont-ils disparaître ? (2) exposé que seul pouvait écrire le savant auquel la science est redevable de tant et de si importantes con trioutions à la connassance des glaciers.

A côté de considérations diverses tendant à établir que, s'il y a actuellement des variations de longueur dans les glaciers ce sont des variations périodiques antour d'un état de longueur moyenne, généralement invariable, considérations qui sont surtout l'expression d'une impression personnelle, $M$. Forel produit à l'appui de sa thèse un argument indirect auquel il attache, avec raison, beaucoup d'importance.

Cet unique argument positif invoqué par l'éminent glaciologiste de Lausanne est l'invariabilité du climat qui aurait été constatée depuis l'époque des palaffites dans les régions préalpines, d'ò̀ M. Forel conclut à une invariabilité analogue

P. Girardin, Observations glaciaires dans la Haute-Maurienne, Ins Grandes-Rousses et IOisans $\left(A n n\right.$. C. A. F., $29^{\prime \prime}$ annee, et Ann. S. T. D., t. XXVIII, 19oz, paru en 1903).

G. Flusin, Ch. Jacob et J. Offner, Rappont pretiminaire sur des observations glaciologaques faites en 1903 (La Geographie, 15 novembre 1903).

Id., Rapport détaillé (sous presse) (Ann. C. A. F. ct Ann, S.T, D., $1903(1904)$.

W. Kilian, Sur lavenir des glaciers dauphinois (B.S. G., $4^{0}$ serie, t. III)

On devra aussi se reporter aux remarquables articles publiés sous le nom de 4 Revues de glaciologie " par M. Rabot Ann. C. A. IN, $28^{\circ}$ et $29^{\circ}$ vol., 1901 (1902) et $1902(1903)$.

(1) Voir à ce sujet les recherches de M. Hauthal, resumés par M. Rabot, (La Nature, igo3).

(2) Il aurait peut-être mioux valu dire: Les glaciers des Alpes disparaítront-ils un jour? personne nayant jamais considéré comme imminente la déchéance de lenscmble des glaciors alpns. 
du climat des hautes régıons. Il est possible, en effet, que dans les Alpes sursses et dans les Alpes centrales, qui ont conservé leurs forêts, le régıme des précipitations atmosphériques n'ait subi aucun changement notable depuis l'époque néolithique. Mais il semble n'en être pas de même dans les Alpes dauphinorses et dans les montagnes du haut bassin de la Durance. M. David-Martin a depuis longtemps déjà attıré l'attention sur la "marche rétrograde de la iégétation forestière " dans les Alpes dauphinoises ; nous arons rappelé à plusieurs reprises, en insistant sur sa réalité et en citant de nouveaux exemples, ce curieux phénomène, que beaucoup de botanistes persistent à attribuer à l'mintervention de l'homme ou des troupeaux, malgré les exemples précis cités à l'encontre de leur thèse. Il est, en effet, d'observation constante, dans le Briançonnais par exemple, de vorr, dans des régıons escarpées, inaccessibles à l'homme et aux troupeaux, la limite supérieure des forêts marquée par une zone d'arbres morts que ne vient remplacer aucune nouvelle poussée forestière ; en beaucoup de points on, assiste pour ainsi dire à cette disparition de la végétation arborescente dans les hautes régions; en d'autres des souches nombreuses enfouies sous les éboulis constituent seules les témons de forêts aujourd'hui disparues. Il est possible que ce processus ait son point de départ dans le déboisement intense que l'homme a fait subir, à une époque historique déjà reculée, à notre région alpine et qui a eu pour conséquence d'en modifier singulièrement le climat, mais 11 semble hors de doute quactuellement cette marche rétrograde de la végétation forestière alpine dans les Alpes dauphinoises se soit transformée en un phénomène naturel continu, qui se poursuit avec une nexorable régularité en dehors de l'intervention humaine.

Ainsi se produit et s'accentue lentement une modification du climat alpestre sans que ses effets soient encore devenus sensibles dans les régions préalpines, mais cette évolution précipite d'une façon toute particulière la déchéance des glacıers dauphinois par la diminution d'humidité qu'elle occasionne.

S'il est difficile de rien dire d'absolu sur l'époque précise où se produira la disparition définitive des glaciers alpins, il est néanmoins très légitume de constater la réduction très rapide de certains glaciers du Dauphiné ; nous pensons pouvorr maintenant, sans craindre aucune objection péremptoire, crorre que " cette déchéance se manifestera cn premier lieu pour les apparells glaciaires les moins importants et les moms alimentés de la chaîne ", en particulier pour ceux $\mathrm{du}$ Dauphiné et de la Haute-Ubaye, dont quelques-uns ont déjà atteint, d'après nous, la dermère phase de leur existence. La phase de retrait actuelle - qui n'est pour la plupart des glaciers qu'un épisode "interglaciaire "- peut en effet, à notre ar'1s, amener, si elle se prolonge, la suppression totale d'un grand nombre de petits glaciers dauphinois, mais il serait téméraire d'affirmer qu'ils ne se reconstitueraient pas s'il survenait, dans l'avenır, une glaciation importante, éventualité dont M. Forel n'a pas envisagé, du reste, les conséquences désastreuses pour un grand nombre de localités habitées des Alpes helvétiques.

Ce que nous savons des oscillations des glaciers pendant les périodes géologiques nous donne cependant à penser que, sı la crise actuelle de décrue n'est que passagère, comme le croit le plus grand nombre des glaciéristes, et que prochainement les glaciers, augmentant de nouveau, doivent reprendre en partie leur ancienne étendue, cette crue ne dépassera très probablement pas en amplitude les plus récentes des glaciations de l'époque pléistocène (quaternaire).

\section{$* *$}

Si nous admettons, avec la plupart des glaciologistes de ce temps, l'existonce de variations cycliques de grande amplitude (variations primaires) ou de moindre importance (variatıons secondaires), ces dernières se produisant à l'intérieur des premières et en sens inverse d'elles, et si nous déduisons des documents réunis si patiemment par M. Rabot (I), que les. varuations primarres constituent des phénomènes généraux affectant la totalıté de la surface terrestre, il n'en est pas moins vrai qu'une sére d'influences locales peuvent singulièrement modifier, en en ralentissant ou en en brusquant les effets, en atténuant ou exagérant leur action, la façon dont se manifesteront ces variations, et parfors même en masquer complètement l'unfluence. On est même en droit de se demander s'il ne peut pas se produire dans certaines régions, par sute du déboisement naturel ou artıficiel (voir plus haut), des modifications climatériques capables de créer des conditions exceptionnelles qui, suivant les cas, hâteront ou renforceront l'effel des périodes de crue ou de décrue. - L'exposition et la dispo. sition topographique même de chaque glacier (présence ou absence d'un bassin de réception permettant la formation d'une réserve suffisante de glace) peuvent aussi influer grandement sur la façon dont se traduisent les effets des variations. générales.

Nous conclurons de là que la nécessité s'impose pour chaque région de procéder à une enquête particulière et indiniduelle des appareils glaciaires qu'elle renferme et des condtıons spéciales, climatériques ou topographiques, qui peuvent, pour certains massifs, notablement changer les résultats prévus par une théorie trop générale des variations glacianres.

La diminution d'altitude causée par des siècles de désagrégation et d'érosion n'est évidemment, avec les variations cycliques, pas le seul facteur qui ait causé la disparition des glaciers de certaines contrées montagneuses, et 11 est très légitime d'admettre que celte disparition at eu lieu plus ou moins tôt suivant que les chaînes ou les montagnes en questıon réalisalent des conditions topographiques ou climatériques plus ou moins favorables à la conservation de réserves glacıaıres De même pour l'avenır, on conçoit aisément qu'il existe des massıfs que leurs conditions climatériques ou topographiques prédestinent, - toutes choses (c'est-à-dire les variations cycliques et l'altıtude) égales d'ailleurs, - à voir disparaître plus tôt leurs glaciers.

Parmi nos glaciers, et malgré l'échéance très lointaine de la déchéance de certains d'entre eux, si tant est que la phase actuelle de décrue doive leur être fatale, nous aurons donc à distinguer :

$I^{\circ}$ Des glaciers relativement peu menacés (ex. : glaciers de la Pilatte, des Etançons, etc.);

$2^{\circ}$ Des glaciers menacés de disparaître à brève échéance (quelques années ou quelques dizaines d'années) (ex: glaciers du versant méridional du Pelvoux, glacier Lombard, glaciers de Marinet, etc.).

Les connaissances de plus en plus parfaites que les travaux récents des géologues ont permis d'acquérir sur les varia. tions qu'ont subies depuis leur apparition les glaciers alpins et sur le rôle qu'ils ont joué pendant les périodes géologiques les plus récentes, ne doivent pas être négligées si nous voulons rationnellement étudier leurs vicissitudes historiques ef essayer de déchiffrer l'avenir qui leur est réservé. Il est, en effet, actuellement admis par l'immense majorité des géologues que ce qưon s'est plu à appeler pendánt longtemps la "période glaciaire" ne correspond pas en réalité à une seule et colossale extension des glaces à l'intérieur et aux alentours des Alpes, mais bien à une succession de glaciations séparées par des phases appelies phases interglaciarres

M̄M Penck et Bruckner ont consacré un bel et intéressant travail (2), dont les livraisons se succèdent rapidement, aux

(x) Reque de Glaciologie (Ann. C.A. F., 29 année, p. 432.)

(2) A. Penck et E Bruckner. - Die Alpen im Eiszeitalter (Les Alpes pendant la pérode glaciaire) Leipzig Chr.-Herm. Tauchnitz, éditeur, in- $\delta^{\circ}$ raisin, avec pl. en couleurs et en phototypie, et nombreuses figures dans le texte Igor-igo4. 
"Alpes à l'époque glaciaire "; il est destiné à dissiper complètement les idées erronées qui peuvent encore subsister dans le public scientifique sur cette phase de l'histoire alpine à laquelle nombre de vulgarisateurs parmı les plus autorisés se sont plu, récemment encore, à prêter des caractères quası fabuleux par la soudaineté et par l'intensité avec laquelle seraient apparues dans nos montagnes des masses de neige et de glace absolument énormes et comparables à l'Inlandsis qui couvre aujourd'hui le Groenland.

Les géologues savent, en effet, que c'est sous l'impulsion des auteurs de ce livre et d'un troisième et éminent savant, prématurément enlevé à la science, Léon du Pasquier, qu'ont été publiées depuis une quinzaine d'années, une série de remarquables études sur les "formations fluvio-glaciaires " des et en est séparé par les traces évidentes d'une periode cle creusement des vallées. Il ressort en outre de ces recherches que chaque système fluvio-gaciare correspond à une phase distuncte d'extension des glaciers, à une "glaciation "durant laquelle les glaciers ont stationné en des points que nous indiquent les moraines qu'ils ont laissées, la dépression centrale qu'lls ont abandonnće et, en aval, les nappes d'alluvions que leurs eaux de fonte ont déposées.

A la phase de creusement qui sépare chacun des systèmes fluvio-glaciaires du suivant, correspond une phase "interglaciarre ", pérode de retrait pendant laquelle les glaciers se retiraient souvent assez loin dans l'intérieur de la chaîne, lasssant, en reculant aınsı, le champ libre à l'action érosıve de leurs eaux de fonte qui approfondissaient les vallées dans

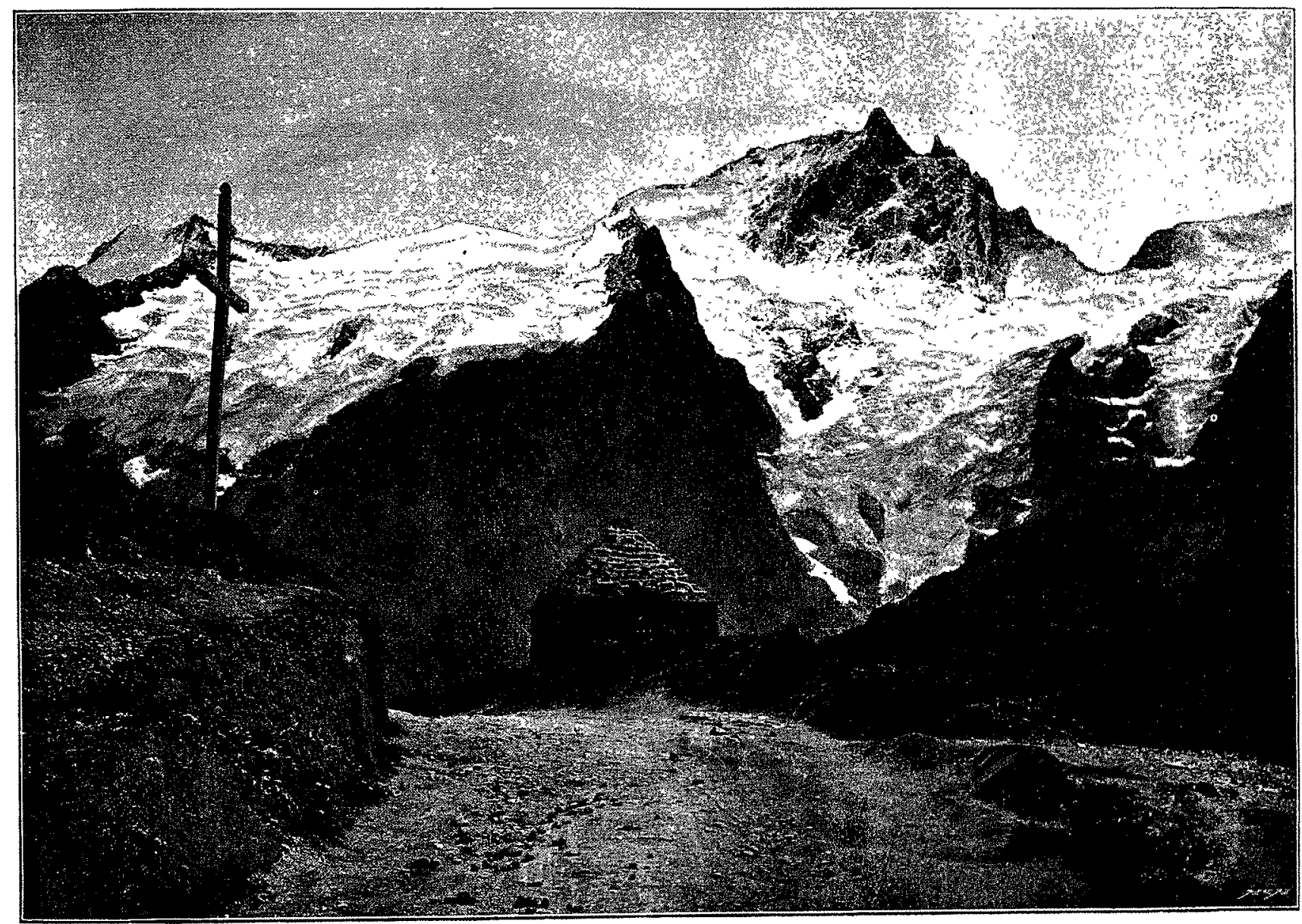

La Meije : 3987 mètres.

Alpes, dans lesquelles était pour la première fois mise en lumière la liaison des "terrasses " d'alluvions anciennes, si développées dans nos grandes vallées alpmes et dans les plaines qui bordent les Alpes, avec les moraines des anciens glaciers. Les premières sont découpées dans des nappes de cailloutis formées par de grands cours d'eau qu'alimentaient les glaciers dont les secondes occuparent la régıon frontale ; en suvant vers l'amont chacune de ces terrasses, on aboutit invariablement à un rempart morainique au delà duquel une dépression en amphithéâtre (dépression centrale ou "cuvette terminale ") marque l'emplacement d'un glacier, aujourd'hu disparu, qui formait la source des courants producteurs d'alluvions.

En appliquant cette méthode à différentes régions des Alpes, MM. du Pasquier, Penck et Briickner ont pu démontrer l'existence d'une série de systèmes fuwio-glaczaires, comprenant chacun une nappe d'alluvions et une suite de moraines indiquant un long stationnement des glaciers. Chacun de ces systemes est en quelque sorte emboâté dans le précédent lesquelles la glaciation suivante devait ramener glaces et moraines.

On a conservé le nom d'Epoque glaciaire à la période assez longue qui comprend cette série de glaciations et d'épisodes interglaciarres et durant laquelle les glaces, d'abord répandues à une altitude considérable au-dessus des vallées actuelles, s'encaissèrent peu à peu, lors de leurs retours successifs, dans ces vallées qui s'approfondissaient toujours par des creusements réltérés.

La succession des phénomènes semble avoir été la même sur le versant Nord des Alpes autrichiennes et bavaroises, on Surse, dans les Alpes italiennes et françaises (I), ainsi qu'il résulte d'une série de monographies locales publiées par MM. Penck, Brickner, du Pasquier et par leurs élèves, mais aucune synthèse détaillée n'avait encore retracé à nos ycux unehis.

(r) Kilian et Penck. - Des dépôts glaciaires et fluvio-glaciaires du bassin de la Durance. $C . R$ Ac. des Sc., 17 juin 1895. 
toire complète de la chaine alpine pendant cette phase intéressante de son historre ( $\mathrm{I}$ ).

C'es glaciations successives, dont l'existence est admise actuellement par tous les observateurs sérieux, peuvent trouver leur explication dans des déplacements de la limite in férieure des nelges persistantes dans les Alpes Beaucoup de chaînes extérieures montrent, en effet, des traces indéniables de leur anczenne couverture de glace. Il est important et curieux de rechercher avec les auteurs et, dans toute l'étendue du massif alpun, combien de fors et dans quelle mesure s'est déplacée la limite inférieure des neiges et comment l'action des neiges et des glaces a modifié, dans le temps et dans l'espace, le relief et les formes topographiques sous-jacentes.

Une série de questions importantes et encore imparfaitement résolues se rattachent du reste à ces recherches; c'est ainsi que le "surcreusement" des grandes vallées, l'érosion glaciaire, la formation des lacs subalpins, le déplacement de certains cours d'eau, l'existence de mouvements du sol à l'époque plérstocène, s'éclairent d'un nouveau jour à la lumière de la méthode employée par MM. Penck et Bruckner.

En reconstituant l'historre des anciens glaciers alpins et en délimitanc les aires qu'ils ont successivement occupées, ces savants nous donnent l'explication d'une foule de particularités topographiques de nos montagnes. Ils nous font assister ainsi à l'évolution du réseau hydrographique et des formes orographiques que nous avons aujourd'hui sous les yeux, en même temps qu'ils nous permettent de comprendre les causes du travail gigantesque et sans cesse renouvelé qui s'est accompli dans la chaîne alpine depus la in de l'époque pliocène, cuvre de démolition et de dénivellement qui se continue encore aujourd'hui et dont aucun alpiniste intelligent n'a le droit de se désintéresser. - On verra en parcourant le beau hvre de MM. Brückner et Penck, qu'une fois encore, le passé s'explique tout naturellement par le jeu de phénomènes identıques à ceux qui s'effectuent actuellement sous nos yeux, et dont il suffit de supposer que l'intensité a été plus grande pendant l'époque pléistocène, sans qu'il y ait lieu de faire intervenir des actions exceptionnelles ou des catastrophes soudanes qu'affectionnaient les anciens naturalistes.

Ajoutons qu'à notre avis, et comme nous l'avons montré ailleurs (2), les sortes de paliers qui, dans beaucoup de vallées alpines (ex.: Durance à la Vachette, la Valloirette à Valloire, etc.), alternent avec des parties plus inclinées et encaissées, ont leur origine dans les stationnements des glaciers pendant les différentes glaciations et dans l'érosion régressive qu'ont exercée successivement les eaux de fonte en avant de ces stationnements.

L'altitude maxima des paliers qui caractérisent la plupart des vallons affluents et même le cours supérieur de nos grandes rivièresl alpines peut être considérée comme indiquant la cote maxima qu'aient atteinte les phénomènes de recul interglaciaires. Il arrive souvent que dans une même vallée il existe plusieurs nuptures de pente et piusieurs de ces paliers correspondant à des creusements interglaciaires successifs d'âges différents.

Plus ces ruptures sont anciennes, plus le travail ultérieur en a atténué les traces (ex.: gorge d'Asfeld à Briançon), qui sont, du reste, appelées à disparaître lorsque les cours d'eau auront réalisé leur courbe d'équilibre.

Ces phénomènes se produisent du reste encore de nos jours. $C^{\prime}$ 'est ainsi que le glacier de la Meije donne naissance à un

(1) Sauf une remarquable, mais très succincte brochure publiée en 1894 par Penck Bruckner et du Pasquier. Le système glaciaire ( $B$ ull. Soc. Nat. de Neufchatel, t. XXII, IS93-94).

Voir en outre. A. Pinck und Richter. Glazialexcursion in die Ostalpen (Liz'ret-Guide du $T^{\mathrm{e}}$ Congrès géologique international, Vienne 1903, fascicule XII.)

(2) Bull. Soc. géol. de France, t. XXVII (rgor) et Ann. Univ. de Grenoble, XXIII, t. no III. torrent qui, depuis que le front de ce glacier s'est retıré en amont de Chalvachère, abandonnant l'ancien thalweg glaciaire, s'est creusé une gorge étroite en entamant profondément ce thalweg granitique et sous la seule action de l'érosion régressive.

En ce qui concerne les Alpes dauphinoises, l'examen géologique des dépôts fiuvio-glaciaires du Bas-Dauphiné, du Graisivaudan, des environs de Grenoble et de la vallée du Drac (I), a permıs de retrouver d'ores et déjà d'une façon certaine les traces de plusieurs glaciations successives dont la plus ancienne poussart le front de ses glaciers jusqu'à Lyor (Croix-Rousse), Vienne et Faramans, et dont les survantes, de moins en moins étendues, ont laissé des témoins de leurs stationnements à Saint-Quentin, Rives, Châbons, à NotreDame-de-l'Osıer, à Rovon, puıs aux Alberges près Urıage. II semble également démontré que, dans notre région, ces phases ont été séparées par des périodes de recul ou phases anterglaciaires.

Nous retrouvons ainsi dans le passé les variations cycliques dẹs glaciers présentant une série de retours successifs dont l'ensemble accuse néanmoins une résultante nettement régressive.

Les oscillations géologiques dont il vient d'être question sont-elles comparables aux var1ations promcrives dont nous avons parlé plus haut à propos des glaciers actuels ou appartrennent-elles à un ordre plus élevé englobant ces dernières : C'est là un problème dont la solution présenterait le plus grand intérêt ; lorsque nous aurons retracé jusque dans tous ses détails l'histoire de nos glaciers depuis leur primitive apparition jusqu'à l'heure actuelle, 1 l sera sans doute possible d'er prévoir, mieux qu'on ne le fait en ce moment, le sort futur ; après avoir éclairé le passé à la lumière du présent, nous pourrons alors, à l'aide du passé, essayer de prévoir l'avenir.

On vient de voir que les données les plus précises de la géologie moderne indiquent que l'époque pléistocène (quaternaire) a vu se produire une série (au moins 3) de glacrations séparées par des phases inlerglaczaires, pendant lesquelles le retrait des neiges et des glaces atteignait des proportions comparables à celles qu'll présente à l'époque actuelle ; on a même distingué, à l'intérieur de ces glaciations, des variatıons moins considérables ou stades.

Quatre ordres de variations dans le sens positif ou négatif ont été distinguées dans les oscillations des glaciers ; les unes ( 1 et IX), aux époques géologiques, semblent avoir correspondu à de très longues durées, probablement uni ou même pluriséculaires, tandis que les autres. (III et IV), constatées par des observations historiques, s'effectuent en des temps plus courts et sont caractérisées par des déplacements du front des glaciers dont les plus forts (variations primaires), ne dépassant pas un seul ou un très petit nombre de kllomètres, ne sont pas comparables avec les oscillations de jo et Ioo kilomètres qu'accusent les glaciations pléistocènes.

\section{A. Epoques géologiques:}

I. Glaciations et phases interglaciaires.

II. Stades (Schwankungen).

\section{B. Période historique:}

III. Variations primaires.

IV. Variations secondaires.

Il est facile dès lors de saisir la nature du probléme.

Faut-il considérer les variations du groupe B (primaires et secondaires) comme l'équivalent et la continuation, sous une forme atténuée et plus brère, des variations du groupe A (glaciations et stades), dont l'amplitude se serait modifiée et

(r) Consulter les travaux récents de MMl. Depéret, Kilian, P. Lory et Hitzel. 
la durée singulièrement restreinte par suite de changements dans les conditions climatérıques ou par l'effet de toute autre cause encore inconnue, ou faut-1l penser que nous nous trouvons réellement en présence de quatre ordres de varıations cycliques s'étant toujours produites ou se produsant encore concurremment et se subordonnant en quelque sorte les unes aux autres; en d'autres termes les variations secondarres étant, comme nous le savons, de simples accidents dans le cours des variatıons primaires, ces dernières ne seraient-elles que des oscillations de détail de stades positifs ou négatıfs ("Schwankungen ") d'ordre supérieur qui, a leur tour, seraient des variations accessorres dont les effets masqueraient des variations de premier ordre (de très grande durée et d'amplitude considérable quoique de moins en moins fortes, ainsi que le montre l'historre du passé), les glaciations et les phases interglaciaires?

La réponse à ces questions ne paraît pas pouvoir être donnée dans l'état actuel de nos connaissances.

Dans la première de ces hypothèses, les glaciers de nos Aípes, après une suite de récurrences offensives, de moins en moms accentuées, tendraient à disparaître dans un avenir relativement court (quelques siècles pour les plus grands d'entre eux, moins encore pour beaucoup d'autres).

Dans la seconde hypothèse, les glaciers alpins se trouveratent en ce moment dans une phase interglaciaure, accidentée par le jeu des variations primaires et secondaires ; cette phase pourrait se prolonger encore quelque temps, entraînant la disparition momentanée d'un certain nombre de petits glaciers (comme beaucoup des glaciers dauphinois) mais serait suivie, dans un avenir plus ou moins élorgné, d'un mouvement général de crue (stade positif ou glaciation) toujours masqué par des variations de détail dont les crues dépasseraient les décrues en importance. Ajoutons que vraisemblablement toutefois, cette nouvelle glaciation serait moins considérable que la dernière glaciation préhistorique.

Quelle que solt celle de ces solutions que les progrès de nos connaissances rendront probable, on vort qu'll s'agit d'échéances trop éloignées pour que la question ainsi posée pusse intéresser, soit ceux qui exploitent, comme les hôteliers et les guides, l'attrait qu'offre aux touristes la parure glacée de nos montagnes, solt ceux qui utilisent, comme les agriculteurs ou les industriels, les cours d'eau et les forces hydrauliques dont les glaciers assurent la continuté et l'abondance du débit, notamment pendant les mois de sécheresse.

Pour ces derniers, en effet, l'intérêt est plus immédiat ; que la phase actuelle de décrue doive être suivie d'une crue primaire ou secondaire, peu leur importe ; ce qu'ils désirent c'est de savoir si une continuation possible pendant quelques dizaines d'années de cette crise de régression peut, dans la région dauphinoise, entraîner la disparition complète, quoique momentanée, de certains de nos glaciers et, dans l'affirmative, quels sont ceux de nos appareuls glaciaires qui échapperont le plus longtemps ou même entièrement à cette déchéance, grâce aux "réserves " de glace de leur bassin d'alimentation. 11 est possible, malgré l'imperfection des données fournies jusqu'à nos jours par les observations glaciologiques faites en Dauphiné, de distinguer dès à présent : $I^{\circ}$ ceux de nos cours d'eau auxquels l'existence, dans leur bassin d'alimentation, de glaciers à " réserves " importantes assure jusqu'à nouvel ordre un débit régulier, $2^{\circ}$ ceux qu'une alimentation par des glaciers se trouvant dans un stade de régression trop avancé et réduit à des plaques de glace sans profondeur ou à de simples névés, menace d'une fâcheuse inégalité de régime ou d'une progressive diminution de débit, $3^{\circ}$ et ceux qu'entretrennent uniquement les neiges et les pluies reçues par la surface plus ou moins étendue de leur bassin de réception.

Ce n'est cependant que lorsqu'on possédera dans ses détails l'histoire complète de l'évolution des glaciers alpins et la marche qu'ont survie, dans le passé, leurs oscillatıons grandes ou petites, que l'on pourra, semble-t-il, émettre sérieusement des pronostics quelque peu probables sur leur avenir et sur leur allure plus ou moins rapidement régressive que parassent cacher à nos yeux leurs oscillations périodiques (I).

\section{***}

La longue suite d'efforts dont nous venons de relater la continuité poursuivie pendant 13 ans nous a appris, on vient de le voir, bien des choses nouvelles sur le régime de nos glaclers, mais elle nous a montré aussi bien des lacunes dans nos connaissances.

Au point de vue scientifique il serait désirable qu'un réper $r$ toire complet des glaciers et des champs de névés pérennes de quelque importance existant actuellement en Dauphiné fût dressé et publié le plus tôt possible, et qu'on s'attachât à noter les variations de chacune de ces unités en comparant soigneusement ces évolutions, individuelles entre elles et avec les variations des grands glaciers, bien observés et surveillés, de la Suisse et des Alpes orientales ; de cette façon seulement 1 sera possible de dégager l'influence des conditions climatér1ques et topographiques locales et l'évolution de nos apparels glactaires, et d'en tirer des pronostics quelque peu ratiomels sur le sort qui leur est réservé.

Des recherches physiques, relatives aux prinemònes thermiques et molécularres qui s'y produisent, seraient également à souhaiter.

Au point de vue de la répercussion de ces études sur la connaissance pratique de nos cours d'eau de montagne producteurs d'énergie, une plus grande précision serait absolu. ment indispensable dans les observations glaciologiques, afm d'arriver, d'une part à un cubage précis des réserves glacianres actuellement existantes dans nos montagnes, et de l'autre à une évaluation précise de l'enneigement anmucl et de ses variatıons, ainsi que des déplacements possibles de la limite des neiges persistantes.

II est' impossible néanmoins de se dissimuler que la réalisation de tels résultats exigerait un personnel nombreux, spécialement préparé et consciencieux, des ressources pécuniarres considérables permettant de créer un outıllage suffisant, de procéder à des levés et des mensurations de précision très étendus et d'installer des apparells nivométriques moomparablement plus précis et plus satisfaisants que ceux dont on s'est contenté jusqu'icr. Une comparaison attentive des relevés nivométrıques dans les hautes régions, avec les observations pluviométriques des stations inférieures, pourrart en particulier être féconde et permettre de découvrir des relations qu'il sarait utile de connârtre ; ces résultats intéresseralent non seulement le régime des torrents et rivières alimentés par des glaciers, mais celui des cours d'eau bien plus nombreux qu'entretiennent presque exclusivement les produits des précipitations atmosphériques.

(I) En ce qui concerne les cours d'eau alpins - et ils sont nombreux - que n'alimente aucun appareil glaciarre, une étude intéressante et féconde, sans doute, en résultats pratiques consisterait à mettre en évidence, notamment par des recherches nivométriques plus précises qu'on ne les a faites jusqu'à ce jour, l'influence que peuvent avoir, on outre, sur le débit des cours d'cau alpins et sur les variations de ce débit l'altitude des bassins de réception, leur étendue exacte, leur topographie et leur constitution géologique (perméabilite du soussol) Ces facteurs peuvent se combiner avec le facteur ahute de façons diverses et produire des résultats parfois très différents, suvvant le rôle dévolu à chacun d'eux. L'extrême difficulté d'evalucr d'une façon réellement scientifique ces diverses influences dans les cas particulicrs, généralement complexes, qui se trouvent réalisés dans la nature, a jusqu'à présent découragé los charcheurs qui s'étaient engagés dans cette voie. Ce n'est, à notre avis, que par une: suite de monographics locales, consacrées chacune au bassin d'un seul cours d'eau, que l'on peut arriver à dégager les lois qui règlent ces phénomènes. 


\section{APERCQU SUR LA RÉPARTITION DES GLAGIERS EN DAUPHIHÉ}

Les glaciers de notre région sont d'importance et de grandeur très variées ; ils appartiennent à des types assez différents ; il est utile de les passer rapidement en revue et d'ind̆iquer en outre le rôle qu'ils jouent au point de vue de l'alimentation de nos principaux cours d'eau.

A. Les glaciers de la chaîne de Belledonne sont peu nom breux, relativement peu importants et possèdent le type des " glaciers de cirques " ; on n'y rencontre pas de glaciers à long cheminement descendant dans les vallées. Nous citerons principalement les glaciers de la Sitre et de Freydane, les névés voisins des pics de Belledonne et, plus au Nord, le glaє1er de Combe-Madame, le Grand Glacier (dit aussi Grand Glacier du Gleyzin) et quelque:j névés insignifiants. Sur le versant $E$ de la même chaîne, une série de glaciers du même type (glaciers du Puygris, de Cléraus, du Grand-Gleyzın, de Villonet, etc.) sont tributaires de l'Arc par le torrent des Villards.

B. Les glaciers des Grandes-Rousses, également limités aux partıes élevées du massif, sont plus considérables : sur le versant occidental, le glacier des Rousses et le glacier de la Cochette; sur le versant oriental, les glaciers de Saint-Sorlin, rersant ses eaux en Savore, et plus au sud, les glaciers des Quirlies, du Grand-Sablat et de Sarennes, tributares du torrent du Ferrand, affluent de la Romanche.

Ious sont des "glaciers suspendus " ou des "glaciers de Erques ", et aucun ne descend dans le fond des vallées.

C. Dans le voismage des Aiguilles-d'Arves, le petit glacier Lombard, entre ces dernières et la cıme de Goléon, est en voie de réduction; nous mentionnerons aussi les névés des TroisEvêchés (versant Nord, tributaires de la Savoie) et quelques autres taches plus ou moins pérennes du massif de la GrandeManche au Nord de la Guisane.

D. Citons aussi le glacier du Galibzer qui s'écoule vers le Nord-Est en Savoie et qui, bren que de forme classique el ayant présenté autre fois une portion encaissée dans un vallon et se termmant non' loin des Mottés par une région frontale avec vallum morainique important, est rédurt actuellement à un petit "glacier de crrque " et à des névés fort restremts.

E Le massif du Pelvoux est le plus riche en appareils glacraires ; ces dernieis y présentent des types assez différents, survant les parties du massif auquel ils appartiennent ; 1ls constituent encore, malgré leur déchéance, un ensemble imposant et une source considérable d'énergie hydraulique.

Dans la partze Nord, on remarque l'énorme plateau glacé du Mont-de-Lans, type spécial, avec sa vaste dépendance le glacier de la Girose, qui envoient vers la gorge de la Romanche nne série de petits "glaciers suspendus", puis les glaciers du Lac, du Vallon, de la Meije, du Tabuchet, du Bec, de Selle-Vieille et de l'Homme, du Lautaret et d'Armande, les glaciers Claire et des Bichettes, plus en pente et plus accidentés. Dans le fond de la vallée d'Arsine, le glacier de la Platedes-Agneaux est un beau et grand "glacier encaissé ". Citons aussi le glacier d'Arsme, enfin les "glaciers suspendus" de Chamoissière, des Agneaux, le glacier supérieur des Cavales, le glacier du Clos-des-Cavales, le glacier supérieur des Agneaux, le glacier de la Casse-Déserte, les glaciers de la Roche-Faurio et de Tombe-Murée.

A l'Est et au Sud-Est, nous trouvons les glaciers du Casset, du Pré-des-Fonds, du Monestier, de Séguret-Foran ou de I'Eychauda, tous quatre à partie inférieure encaissée, mais réduits actuellement à leur tronçon supérieur ; le glacier de Séguret-Avant (suspendu), le glacier de la Pyramide ; enfin les glaciers Blanc et Noir, fleuves de glace autrefois confluents ; les "glaciers suspendus " de la Momie, des VioJettes, du Clos-de-l'Homme, le glacier Sans-Nom, le glacier du Coup-de-Sabre, les glaciers du sommet du Pelvoux et des Ecrins ; les grands glaciers d'Ailefroide et du Sélé (à langue terminale s'étalant au pied d'un cirque grandiose), le glacier des Bœufs-Rouges, etc.

Mentionnons encore, à l'ouest de Vallouise, les glaciers du Loup et de Sellar ; enfln les petits névés de l'Eyglière.

Dans le centre, le bassin du Vénéon avec ses tributaires, le ruisseau du Vallon-des-Etages, le ruisseau des Etançons et le Haut-Vénéon, offre un ensemble plein de grandeur et des plus remarquables de glaciers importants, dont un grand nombre sont des "glaciers de vallée ", encaissés dans leur partie inférieure ; parmi les plus grands il y a lieu de citer: les glaciers du Vallon-des-Etages, du Chardon, de la Pilatte, de la Temple, de la Bonne-Pierre, des Etançons, de la Selle. Il existe, en outre, dans ce bassin, une série de glaciers plus petıts, pour la plupart suspendus ou tributaires des glaciers de vallées susmentionnées ; ce sont : le glacier du Soreiller, le glacier du Plat, le glacier du Routier, le glacier du Burlan, les glaciers de la Gandolière, du Plaret, le glacier de la Grande-Rume, les glaciers de la Somme, d'Alvau, de la Vera-Pervoz; le glacier du Vallon-de-la-Pilatte, le glacier de la CosteRouge, le glacier Long, le glacier Gris ; le glacier du Coin (tributaires du glacier de la P1latte), le glacier du Says (1d.), le glacier de Baverjat, le glacier de Chéret, le glacier de l'Encoula, le glacier de l'Ours, le glacier des Fétoules, le glacier de I'Etret, le glacier de la Lavey, le glacier des Roures (tributare du glacier du Chardon), le glacier du Fond de la Muan de, le glacier des Sellettes.

A l'Ouest, dans des montagnes qui, au point de vue géologique, dorvent être considérées en partie comme la continuatıon méridionale de la chaîne des Grandes-Rousses au Surl de la Romanche, mais qui appartiennent à la partıe occıdentale du bassin du Vénéon, une série de glaciers notables attrent l'attention. Ce sont les glaciers de Villard-Eymond, de la Muzelle, le glacier du Vallon-de-Lanchâtra, les glaciers de Montagnon, du Vallon, les glaciers du Pierroux, de la Mariande et des Arias, du Lac, d'Entrepierroux, etc.

Le versant mérzdional du massif du Pelvoux, quoique des traces très fraîches $y$ attestent, d'une façon très évidente, l'existence de glaciers d'une certaine importance à une époque assez rapprochée de nous, n'offre plus que des " glaciers suspendus " à pente assez raide, tels que les glaciers de la brèche de Valenestre, les glaciers de la Haute-Pisse, le giacier an Sud-Ouest de la Muzelle, le glacier Court, le glacier de l'Aigulle-d Olan, le glacier Courbé, le glacier de l'Aigulle-Rousse, le glacier de Coin-Charnier ou des Marmes, les glaciers du Grand-Vallon et du Petit-Vallon, le glacier du Fond-de-Tursat, le glacier du Clot, le glacier d'Olan, le glacier du Vallon de la Rouye, le glacier de Chalance, le glacier du Lauzon, le glacier de Gioberney, le glacier des Aupilloux.

Une partie d'entre eux accusent une grande réduction, plusieurs semblent à la veille de disparaittre ; les glaciers de Porteras et de la Grande-Roche ont cessé d'exister. - Citons aussi le glacier des Souffles (ou du Dévoluy), suspendu et très réduit.

Flus au Sud encore, entre le Drac et le Valgaudemard, les glaciers de Chaillol, de Malcros, les névés de Val-Estrèche, les glaciers et névés de Crupillouse, actuellement insignifiants, et les glaciers du Sirac (glacier de Verdonne, etc.) n'ont qu'une médiocre importance : aucun d'eux ne présente le. "type encaissé ".

$\bar{F}$. Enfin, tout à fait au Sud, dans le haut de la vallée de Freyssinières, sur les flancs Nord du Grand-Pinier, un cirque rocheux abrite le petit glacier de Chaulier, le plus méridional du massif.

G. Bien que n'appartenant plus à la région dauphinoise proprement dite, la haute vallée de l'Ubaye mérite d'être citée icl comme possédant, dans son bassin, les glaciers de Marinet, les plus méridionaux des Alpes françaises ; nous avons fait voir en détail ailleurs, la régression rapide qui a frappé depuis quelques années ces glaciers, restes actuellement peu importants d'appareils glaciaires, jadis puissants et étendus. 


\section{$*^{*} *$}

11 est facile de distinguer parmi les glaciers cités dans cette énumération un petit nombre de types bien caractérisés entre lesquels se répartissent tous les glaciers dauphinois. Ce sont d'abord ceux dont le vaste bassin d'alimentation, en forme de cuvette ou de cirque où s'accumulent d'épaisses réserves de glace, est suivi d'une région de cheminement relatıvement longue et encaissée ; ces glaciers de vallées se terminent généralement par une région frontale étalée en éventa1 en arrière d'un ou de plusieurs vallums moramiques. Les glacters de la Pilatte et du Chardon constituent de bons exemples de ce type, dont la région frontale est souvent, du reste, encombrée de matériaux morainiques.

Beaucoup de glaciers de vallées ne sont plus représentés souvent, par suite de la décrue prolongée qu'ils ont subie, que par leur bassin d'alımentation, sorte de culot de glace occupant le fond d'un cirque rocheux, la région de cheminement ayant été presque entièrement détruite par l'ablation ; ce sont des glaciers de cirques, comme les-glaciers de Freydane, du Casset, des Etançons, etc.

Enfin, un type très répandu consiste, soit en plateaux ou cuvettes glacés ou en sortes de plaques de glace sur des pentes abritées, sans région de cheminement individualisée. Ces glaciers suspendus, très fréquents dans les Alpes dauphinoises, se présentent sous deux formes principales : la premère comprend des glaciers très importants, possédant d'épaisses réserves de glaces comblant des cavités importantes et dont la situation topographique seule a empêché l'écoulement dans une vallée ; les glaciers du Mont-de-Lans, de la Grose, des Rousses, des Quirlies en sont des exemples remarquables ; la deuxième forme consiste en plaques ou taches de glace occupant certaines parties protégées des pentes montagneuses, certaines anfractuosités, de petits cirques et des paliers rocheux ; cette variété ne représente en somme que des témoins, épargnés par la fusion, de glaciers plus considérables, des lambeaux d'une couverture glacée jadis continue ; c'est le type le plus fréquent dans les régions qui ont subi une décrue glaciaire forte et prolongée. On en voit des exemples surtout sur les versants méridionaux du massif du Pelvoux.

\section{$* *$}

Si maintenant nous examinons la façon dont se répartissent ces glaciers dans l'alimentation de nos principatux cours d'eau, nous remarquons ce qui suit :

L'Isère est, surtout en amont de Grenoble, tributaire des glaciers importants de la Haute-Maurienne et de la HauteTarentaise, dont nous n'avons pas à nous occuper ici.

Les apports qu'elle reçoit, sur sa rıve droite, des torrents descendus de la chaîne de Belledonne (sensu lato), entre Montmélian et Grenoble, ne lui apportent que les eaux de quelques petits glaciers en voie de régression.

Le Drac, si l'on met à part la Romanche, ne possède dans son bassin d'alimentation aucun glacier de premier ordre, riche en réserve pour l'avenir ; ses affluents la Bonne, la Sévera1sse, etc..., lui apportent l'eau de fusion d'une série de petits glaciers de cirque ou de glaciers suspendus (glacier de Gioberney, glacier du Fond-de-Turbat, etc.), ainsi que le Drac de Champoléon qu'aliment en partie les petits glaciers du
Sirac.

La Romanche est la rivière du Dauphiné la plus riche en réserves glaciaires importantes; c'est dans le bassin de ce cours d'eau que se trouvent les glaciers les moins menacés et les plus importants. - Elle se nourrit aux glaciers, grands et petits, de toute la partie septentrionale et centrale du Pelvoux, par son cours supérieur et par le Vénéon et ses affluents; elle recueille en outre les eaux d'une grande partie des glaciers des Grandes-Rousses.

La Durance reçoit, en aval de Briançon, par la Guisane, la Gyronde et leurs affuents, les eaux de fonte des glaciers du
Casset, du Monêtier, de l'Eychauda et surtout celles des appareils glaciaires si importants (glacier Blanc, glacier Nolr, glacier du Sélé) des environs de Vallouise, alimentés par de puissantes réserves.

Le Gual ne compte, dans son bassin, que des névés isolés dans le massif de Font-Sancte et dans les crêtes frontières voisines du Viso (Pointe-Joanne). Ce cours d'eau ne peut donc être considéré comme étant alimenté par des glaciers.

L'Ubaye ne compte parmi ses tributaires que les glaciers de Marmet, peu considérables et en voie de déchéance complète.

On voit donc que, s'il y a quelque intérêt pratique à encourager l'étude des glaciers dauphinois, il serait d'une utilité bien plus grande encore pour la connaissance de nos forces hydraulıques et de leur régime d'instituer et d'entretenir des recherches précisés et méthodiques sur l'enneigement des hautes régions de nos Alpes.

$$
\begin{aligned}
& \text { W. Kitian, } \\
& \text { Professeur à l'Universite de Grenoble. }
\end{aligned}
$$

\section{MESURE DE LA VITESSE D'UN COURS D'EAU}

La mesure de la vitesse d'un cours d'eau, rivière ou canal, a fait et fait encore l'objet de nombreuses études dans diverses Revues. Les moulinets de Woltmann, les tubes de Pitot, les flotteurs ont été mis à contribution avec des succès divers.

Si les moulinets de Woltmann et les tubes de Pitot sont des appareils assez commodes pour l'évaluation de la vitesse de l'eau dans un canal, leur exactitude n'est guère satisfaisante que quand cette vitesse est un peu forte. Pour une vitesse tres réduite les résultats de la mesure sont parfois assez incertains.

L'emploi des flotteurs, s'il est plus commode et à la portée de tout le monde, est sujet à d'autres erreurs; de plus, avec cux, on ne peut apprécier des vitesses à des profondeurs différentes de telle sorte que l'on ne peut avoir qu'une indication sur la valeur de la vitesse moyenne.

M. H4sos, dans le Zentralblatt der Bauverualtung, a cependant proposé d'utiliser ces flotteurs d'après une nouvelle

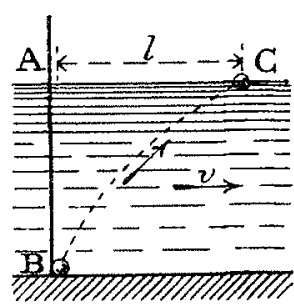

Fig. 1

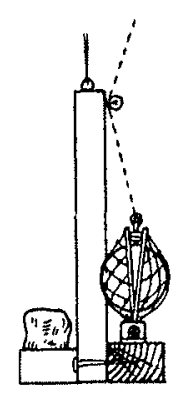

Fig. 2

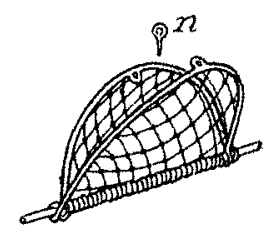

Fig. 3 méthode, que nous reproduisons à titre d'indication, et qui permet d'apprécier la moyenne des vitesses des tilets liquides a differentes hauteurs.

Considérons figure i une boule de faible densité, maintenue au fond de l'cau en un point B. Si l'on abandonne cette boule à elle-même, elle remontera verticalement si le cours d'eau est immobile, c'est-à-dire si $y=0$. La vitesse d'ascension de cette boule sera dautant plus grande que sa densité sera plus faible et celle du liquide plus forte.

Si le cours d'eau est animé d'un certain mouvement, la boule ne montera plus vericalement mais décrira une trajectoire dont la projection horizontale $\mathrm{AC}=$ l serad'autant plus grande que la vitesse moyenne du cours d'eau sera elle-même plus forte. Le quotient $\frac{l}{t}$ peut être approximativement considéré comme la moyenne des vitesses des filets liquides du cours d'eau dans le voisinage de la section $\mathrm{AB}$ et du plan $\mathrm{BAC}$. 